\title{
Low-dose intrathecal local anesthetic does not increase the threshold current for the epidural stimulation test: a prospective observational trial of neuraxial analgesia in labouring women Une faible dose intrathécale d'anesthésique local n'augmente pas le courant de seuil pour le test de stimulation péridurale: étude prospective observationnelle d'analgésie neuraxiale chez des femmes en travail
}

\author{
Jose C. A. Carvalho, MD, PhD • Rakhi Khemka, MD • \\ Julian Loke, MBBS · Ban C. H. Tsui, MD \\ Received: 2 July 2014/ Accepted: 2 December 2014/Published online: 11 December 2014 \\ (c) Canadian Anesthesiologists' Society 2014
}

\begin{abstract}
Purpose The purpose of this study was to investigate the ability of the electrical epidural stimulation test (EST) to determine the position of the epidural catheter during combined spinal-epidural (CSE) anesthesia for labour analgesia.
\end{abstract}

Author contributions Jose C.A. Carvalho, Rakhi Khemka, Julian Loke, and Ban C.H. Tsui provided substantial contributions to study conception and design and analysis and interpretation of data. Jose C.A. Carvalho, Julian Loke, and Ban C.H. Tsui participated in drafting the article and revising it critically for important intellectual content. Jose C.A. Carvalho and Rakhi Khemka contributed to data collection.

J. C. A. Carvalho, MD, PhD - R. Khemka, MD

Department of Anesthesia and Pain Management, Mount Sinai Hospital, University of Toronto, Toronto, ON, Canada

J. C. A. Carvalho, $\mathrm{MD}, \mathrm{PhD}$

Department of Obstetrics and Gynecology, Mount Sinai

Hospital, University of Toronto, Toronto, ON, Canada

J. Loke, MBBS

Department of Anesthesiology, Pharmacology, and

Therapeutics, University of British Columbia, Vancouver, BC,

Canada

J. Loke, MBBS

Abbotsford Regional Hospital and Cancer Centre, Abbotsford, BC, Canada

B. C. H. Tsui, MD ( $\square)$

Department of Anesthesiology and Pain Medicine, University of Alberta, 8-120 Clinical Sciences Building, Edmonton, AB T6G 2G3, Canada

e-mail: btsui@ualberta.ca
Methods This was a prospective observational trial of attempted EST during neuraxial analgesia in labouring women. Ten women received a double-segment CSE technique and one woman underwent continuous spinal analgesia following inadvertent dural puncture and deliberate placement of the catheter tip in the intrathecal space. In all CSE cases, the spinal injection was performed below the level of the epidural insertion. The motor threshold current (MTC) was determined by EST through the existing epidural/intrathecal catheter immediately following and at five, ten, and 15 mins after intrathecal injection of bupivacaine $1.75 \mathrm{mg}$ and fentanyl $15 \mu \mathrm{g}$. Changes in the MTC were expressed as a percent change compared with baseline.

Results The MTC required to elicit muscle contractions in women with epidurally placed catheters was unaffected by the intrathecal injection of the analgesic mixture $(P=0.731)$. The MTC increased following an intrathecal injection of the same mixture in a woman who had the catheter placed intrathecally.

Conclusions The intrathecal injection of a low dose of bupivacaine-fentanyl does not affect the MTC if the catheter is placed in the epidural space; however, it does affect the threshold if the catheter is placed intrathecally. We also confirm that the EST can help to determine the position of the epidural catheter prior to injection of the test dose. This trial was registered at ClinicalTrials.gov (NCT00464841).

Résumé

Objectif L'objet de cette étude était d'analyser la capacité du test de stimulation électrique péridurale 
(ETS) à déterminer la position du cathéter péridural au cours d'une anesthésie combinée rachianesthésiepéridurale (CSE) pour l'analgésie du travail.

Méthodes Il s'agit d'une étude prospective observationnelle de tentatives du TSE au cours de l'analgésie neuraxiale chez des femmes en travail. Dix femmes ont reçu une technique d'ACRP à double segment et une femme a subi une rachianesthésie continue après ponction accidentelle de la dure-mère et positionnement délibéré de l'extrémité du cathéter dans l'espace intrathécal. Dans tous les cas d'une ACRP, l'injection de rachianesthésie a été effectuée en dessous de l'insertion péridurale. Le seuil de courant du bloc moteur (MTC) était déterminé par TSE au moyen du cathéter existant péridural/intrathécal, immédiatement après l'injection intrathécale de bupivacaïne $1,75 \mathrm{mg}$ et fentanyl $15 \mu \mathrm{g}$, puis 5, 10 et 15 minutes plus tard. Les modifications du MTC ont été exprimées en variation de pourcentage par rapport aux valeurs de référence.

Résultats Le MTC nécessaire au déclenchement de contractions musculaires chez les femmes ayant un cathéter péridural en place n'a pas été modifié par l'injection intrathécale $d u$ mélange analgésique $(P=0,731)$. Le MTC a augmenté après l'injection intrathécale $d u$ même mélange chez une femme dont le cathéter était en situation intrathécale.

Conclusions L'injection intrathécale de faibles doses de bupivacaïne-fentanyl ne modifie pas le MTC si le cathéter est placé dans l'espace péridural; toutefois, elle modifie le seuil si le cathéter est placé en position intrathécale. Nous confirmons également que le TSE peut aider à déterminer la position du cathéter péridural avant l'injection de la dose de test. Cette étude a été enregistrée sur le site www. clinicaltrials.gov (NCT00464841).

Combined spinal-epidural (CSE) is an established technique for providing labour analgesia to obstetric patients. $^{1-5}$ The initial single-injection spinal component produces rapid onset but unsustained analgesia. Subsequently, the epidural catheter can be used to extend and provide continuous pain relief. In the context of a conventional epidural technique, the catheter can be tested either by the epidural stimulation test $(\mathrm{EST})^{6,7}$ or by introducing medication via the catheter and determining the clinical effect. During single-segment needle-throughneedle CSE, however, the epidural catheter is initially "untested" - that is, the test dose has not yet been injected through it. The effects of the spinal anesthetic delay the ability to detect a definitive response to drugs in the epidural space.
Table 1 Demographic data for epidural and intrathecal catheter groups

\begin{tabular}{lll}
\hline & Epidural $(n=10)^{*}$ & Intrathecal $(n=1)$ \\
\hline Age $(\mathrm{yr})$ & $33(4)$ & 33 \\
Height $(\mathrm{cm})$ & $165(9)$ & 165 \\
Weight $(\mathrm{kg})$ & $81.3(11.8)$ & 97.5 \\
BMI $\left(\mathrm{kg} \cdot \mathrm{m}^{-2}\right)$ & $29.9(3.6)$ & 35.8 \\
Gestational age (weeks) & $40.4(1.1)$ & 40.1 \\
Gravida/para & $1 / 0$ & $3 / 0$ \\
\hline
\end{tabular}

* Mean (SD), except for gravida/para, which are median values

The EST (also known as the Tsui test) for confirming placement of epidural catheters was first described over 15 years ago, and multiple subsequent original reports have shown success using the technique. ${ }^{8-18}$ The motor threshold current (MTC) achieved when using the EST can distinguish whether the catheter tip is positioned epidurally (medium MTC: 2-15 mA), subcutaneously (high MTC: $>15 \mathrm{~mA}$ ), or intrathecally (low MTC: $<2 \mathrm{~mA}$ ), although a low MTC might also conceivably be due to the catheter abutting a nerve root. It should be emphasized that these MTC ranges are somewhat arbitrary depending on the individual interpreting the EST results. For example, for some, $1 \mathrm{~mA}$ is acceptable as the minimum MTC for a positive result, while for others, $2 \mathrm{~mA}$ may be the minimum. Likewise, maximum acceptable positive MTC values may be $10 \mathrm{~mA}$, $15 \mathrm{~mA}$, or $>15 \mathrm{~mA}$. Despite the proven success of the EST in epidural catheter placement, its definitive anatomical site of action has yet to be shown. The presumed mechanism is thought to be through stimulation of spinal nerve roots and segmental motor neurons rather than by the spinal cord itself.

The EST has previously been applied to obstetric epidurals, but not in the setting of CSE. ${ }^{19}$ Accordingly, in this observational trial, we investigated whether EST can be used to distinguish the position of an epidural catheter in labouring women receiving CSE. We hypothesized that a low dose of local anesthetic injected intrathecally would exert no or minimal effect on the MTC during the EST. Such a result would support the notion that the site of action of the EST is at the level of the spinal nerve rather than at the spinal cord or spinal nerve root.

\section{Methods}

This was a prospective observational trial to assess the utility of the EST in cases where the epidural catheter was placed epidurally or intrathecally. Research Ethics Board approval was obtained from Mount Sinai Hospital, Toronto, ON, Canada in December 2006. Following 
Table 2 Results of epidural stimulation test for patients with either epidural or intrathecal catheter tip placement

\begin{tabular}{|c|c|c|c|c|c|}
\hline \multicolumn{2}{|c|}{ Epidural group } & \multicolumn{4}{|c|}{ MTC (mA) following intrathecal injection (\% change compared with baseline MTC) } \\
\hline Subject & Baseline MTC (mA) & Immediately after & 5 min after & 10 min after & 15 min after \\
\hline 1 & 7 & $7(0 \%)$ & $7(0 \%)$ & $6(-17 \%)$ & $7(0 \%)$ \\
\hline 2 & 4 & $4(0 \%)$ & $4(0 \%)$ & $4(0 \%)$ & $4(0 \%)$ \\
\hline 3 & 5 & $5(0 \%)$ & $4(-25 \%)$ & $6(+20 \%)$ & $6(+20 \%)$ \\
\hline 4 & 5 & $5(0 \%)$ & $5(0 \%)$ & $5(0 \%)$ & $5(0 \%)$ \\
\hline 5 & 4 & $4(0 \%)$ & $4(0 \%)$ & $4(0 \%)$ & $4(0 \%)$ \\
\hline 6 & 4 & $4(0 \%)$ & $5(+25 \%)$ & $5(+25 \%)$ & $5(+25 \%)$ \\
\hline 7 & 10 & $10(0 \%)$ & $9(-11 \%)$ & $11(+10 \%)$ & $10(0 \%)$ \\
\hline 8 & 7 & $6(-14 \%)$ & $5(-28 \%)$ & $5(-28 \%)$ & $5(-28 \%)$ \\
\hline 9 & 6 & $6(0 \%)$ & $6(0 \%)$ & $6(0 \%)$ & $6(0 \%)$ \\
\hline 10 & 2 & $2(0 \%)$ & $2(0 \%)$ & $2(0 \%)$ & $2(0 \%)$ \\
\hline \multicolumn{2}{|c|}{ Average $\%$ change $v s$ baseline current } & $-1.4 \%$ & $-3.9 \%$ & $-1.0 \%$ & $+1.7 \%$ \\
\hline \multicolumn{2}{|c|}{ Intrathecal group } & \multicolumn{4}{|c|}{ MTC (mA) following intrathecal dose (\% change compared with baseline MTC) } \\
\hline Subject & Baseline current (mA) & Immediately after & 5 min after & 10 min after & 15 min after \\
\hline 1 & 1.2 & $>2 *$ & $>2$ & $>2$ & $>2$ \\
\hline \multicolumn{2}{|c|}{ Average $\%$ change $v s$ baseline current } & $>67 \%$ & $>67 \%$ & $>67 \%$ & $>67 \%$ \\
\hline
\end{tabular}

MTC $=$ motor threshold current

* The protocol restricted increasing the MTC above $2 \mathrm{~mA}$

Figure Interpretation of the epidural stimulation test (EST) during combined spinalepidural (CSE). Various interpretations of the EST during a CSE procedure are possible. Following CSE (blue boxes), three different results can occur (yellow boxes). In cases of motor threshold current (MTC), interpretations of the EST can be ambiguous (grey boxes), which may or may not be clarified by aspirating for cerebrospinal fluid (CSF). If aspiration is positive, intrathecal placement is indicated; if aspiration is negative, catheter tip position remains unknown. In cases of intrathecal placement, a low dose of local anesthetic/opioid can be injected through the catheter to achieve anesthesia. In cases of a motor response but negative CSF aspiration, a test dose of local anesthetic can be injected; if the test dose is positive (i.e., rapid anesthesia), the catheter tip is in the intrathecal space. See text for details

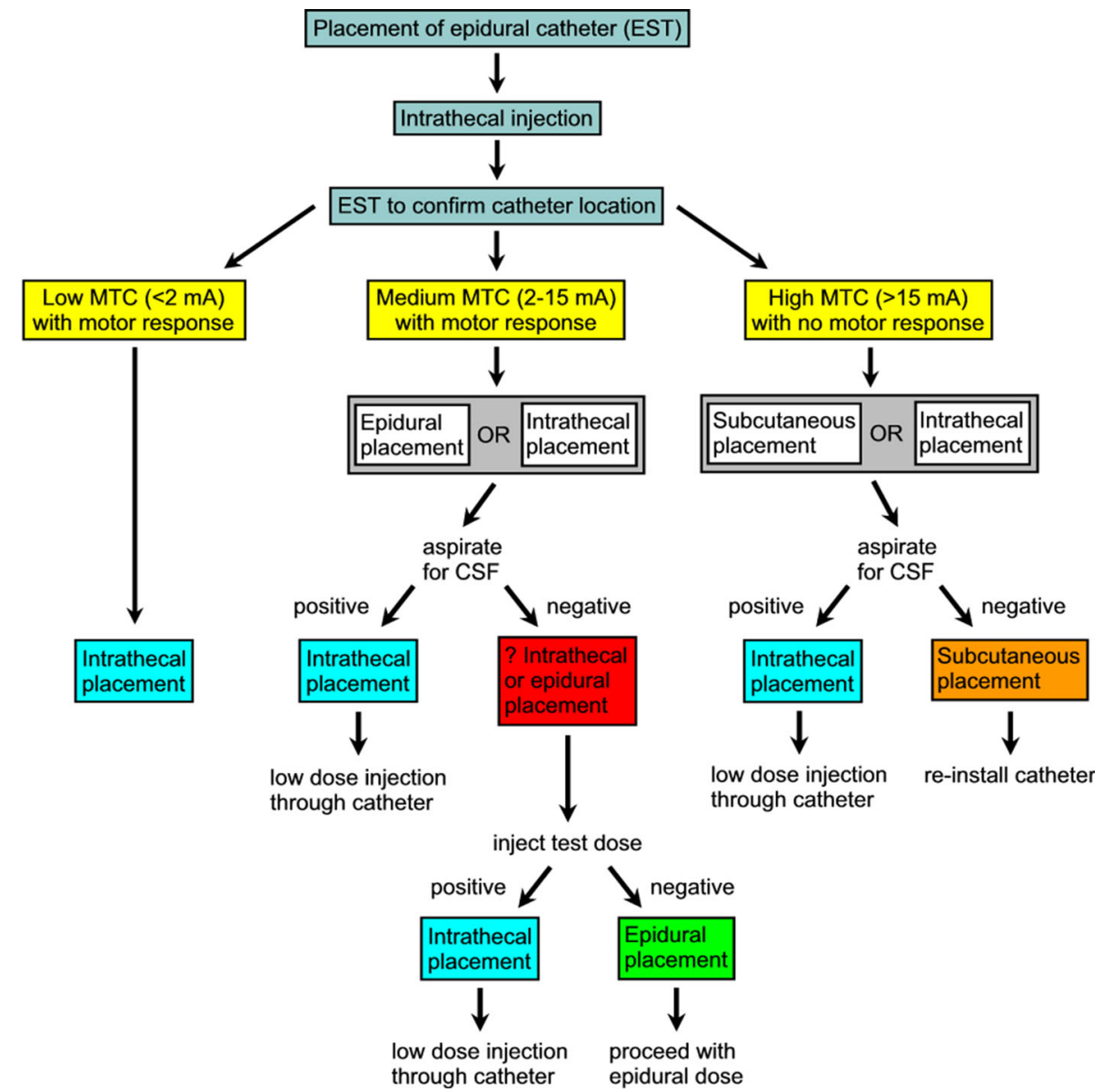


written informed consent, term pregnant women in early labour were recruited into the study. Inclusion criteria were females $18 \mathrm{yr}$ and older, American Society of Anesthesiologists physical status I or II, and full-term pregnancy along with a request for epidural early in labour. Exclusion criteria included a contraindication to regional anesthesia; allergy or hypersensitivity to lidocaine, bupivacaine, or fentanyl; use of sedatives or opioids; abnormal vertebral anatomy; neurological disorder with lumbar involvement; and implanted electronic devices. A sample size of twenty women - ten with properly placed epidural catheters and ten with inadvertent intrathecal catheters - was chosen for convenience.

A double-segment CSE technique ${ }^{1,5}$ was chosen to facilitate performing an EST both before and after spinal anesthesia. Under aseptic conditions, the lumbar epidural space was located using a $17 \mathrm{G}$ Tuohy needle and loss of resistance to air. An Arrow ${ }^{\circledR}$ FlexTip Plus ${ }^{\circledR}$ (Teleflex, Markham, ON, Canada) end-opening catheter, constructed with an electrically conductive spiral wire in contact with the fluid path, was inserted and filled with $0.9 \%$ saline $1 \mathrm{~mL}$. The MTC was then determined by the $\mathrm{EST}^{7}$ using an EZStim II nerve stimulator (Life-Tech International, Stafford, TX, USA). By protocol, the result of the EST was not used as the basis for any clinical decision during the study. The EST was deemed a failure when a motor response was absent at $20 \mathrm{~mA}$ and the epidural turned out to be working clinically. Spinal anesthesia was then performed with a separate $27 \mathrm{G}$ Whitacre needle at the intervertebral level immediately below the epidural insertion site. Following return of clear cerebrospinal fluid (CSF), $0.25 \%$ isobaric bupivacaine $0.7 \mathrm{~mL}$ with fentanyl $15 \mu \mathrm{g}$ was introduced intrathecally, and the needle was then removed. The EST was then repeated through the existing epidural catheter. The MTC was determined immediately following the intrathecal injection and repeated at five, ten, and $15 \mathrm{~min}$ after spinal anesthesia. Changes in MTC were expressed as a percent change compared with baseline. Effective spinal anesthesia was confirmed clinically by demonstration of a sensory block (to ice) and development of effective analgesia.

In the event of an inadvertent dural puncture, a FlexTip Plus catheter was deliberately inserted intrathecally. The MTC was then measured using the EST to a maximum of $2 \mathrm{~mA}$. By protocol, if motor response was absent at $2 \mathrm{~mA}$, the EST was deemed a failure. A dose of $0.25 \%$ isobaric bupivacaine $0.7 \mathrm{~mL}$ with fentanyl $15 \mu \mathrm{g}$ was then introduced through the intrathecal catheter. The EST was repeated, and the MTC was measured immediately following injection and then at five, ten, and 15 min after injection. The study was concluded at the completion of the assessments, and the catheter was used to provide continuous spinal analgesia until delivery.
Statistical analysis

To test whether low-dose intrathecal local anesthetic had an effect on the threshold current of EST over time, we selected the MTC as the dependent variable and intrathecal local anesthetic as the independent variable, with the latter consisting of five groups based on time: 1) no intrathecal local anesthetic, 2) immediately after intrathecal dose, 3) five minutes after intrathecal dose, 4) ten minutes after intrathecal dose, and 5) 15 min after intrathecal dose. No assumption was made regarding normality. The Friedman non-parametric test for repeated measures was selected to test for any differences between groups since the dependent variable being measured was ordinal in this study. All data were analyzed in SPSS $^{\circledR}$ version $20\left(\right.$ IBM $^{\circledR}$, Armonk, NY, USA). All reported $P$ values are two sided.

\section{Results}

Eleven women were enrolled, with only one sustaining an inadvertent dural puncture. After recruiting this subject, we decided to terminate the study due to futility in the intrathecal group because of protocol limitations (see Discussion). Demographic and obstetric data are provided in Table 1. Results are shown in Table 2 and the Figure. All women receiving an epidural catheter required medium MTC consistent with correct epidural position, and there was no statistically significant difference in MTC across the different time points before and after spinal anesthesia $(P=0.731)$. In the single case with an intrathecal catheter, baseline MTC was determined to be $1.2 \mathrm{~mA}$; however, after the intrathecal injection of the analgesic mixture, the MTC could not be determined beyond the protocol limit of $2 \mathrm{~mA}$ (Figure).

\section{Discussion}

This is a novel report to show that an intrathecal injection of a low dose of bupivacaine-fentanyl has a minimal effect on the MTC of the EST with an epidural catheter. In contrast, low-dose bupivacaine-fentanyl was associated with an increase in MTC when the catheter was placed intrathecally; however, precise MTC readings could not be obtained with the intrathecal catheter (Table 2). We were prevented from determining whether an intrathecal injection eliminates the ability to perform the EST due to the protocol limiting the current that could be applied. Nevertheless, our findings support the hypothesis that the site of action of the EST is at the spinal nerve rather than at the spinal cord or the spinal nerve root, which has its own dural cuff. $^{20}$ Nevertheless, further work needs to be done to provide 
definitive proof that this is the case. We have also shown that the MTC of an intrathecal catheter in a term pregnant woman falls within the previously reported range. ${ }^{21}$

Electrical stimulation can be used as an electrodiagnostic test to locate the site of nerve injury. Immediately after a nerve is injured or transected, there is typically absence of an electrical response to proximal stimulation but normal response to distal stimulation to the affected site. ${ }^{22}$ In other words, one would expect a motor response to occur only if stimulation were distal to the site of nerve injury (or blockade with local anesthetic). In this study, we showed normal stimulation of the epidural catheter following an intrathecal injection of a low dose of local anesthetic, thus supporting the theory that the EST acts "distally" to the intrathecal space. Due to the limitations of the study design, however, more research needs to be done to show unequivocally that the site of action is at the spinal nerve.

With the current study design, our patients did not receive an intrathecal dose equivalent to that which would simulate absence of an electrical response to proximal stimulation, i.e., full motor block. Indeed, the intrathecal concentration of bupivacaine, which was already a relatively low dose, may have been even lower around the nerve roots at the level of the stimulation. This is because the position of the catheter tip is unknown and may have been more than one spinal segment away from the intrathecal injection site. Thus, we cannot state with certainty that the EST is acting on spinal nerves and distal to the nerve root. Another limitation of this study is that, with the protocol limit of $2 \mathrm{~mA}$ for intrathecal stimulation, we are uncertain whether a higher stimulus current within the general limits of the EST $(2-20 \mathrm{~mA})^{23}$ would have been sufficient to elicit a motor response through an intrathecal catheter following the intrathecal administration of local anesthetic and opioid.

As shown in the Figure, the lack of motor response in our case with an intrathecal catheter could be consistent with two possible scenarios that could not be examined with this study protocol: one in which intrathecal stimulation at a medium threshold current (2-15 mA) produces a motor response, and another in which a high intrathecal current ( $>15 \mathrm{~mA}$ ) does not produce a motor response. In the former scenario, one may hypothesize that the electrical current passes from an intrathecal catheter to the adjoining epidural space and stimulates the spinal nerve to provoke a motor response. From the data derived from this study, we would argue that such stimulation could occur at a range similar to that for a catheter tip correctly placed in the epidural space. In the latter scenario, the most likely explanation is that, inside the intrathecal space, the electric current would be conducted through the path of least resistance entirely within the electrolyte-rich CSF. This explains the very low threshold current needed to stimulate the anterior spinal nerve root which is bathed in CSF. With the electrolyte-filled dural sac acting as a "lightning rod", the density of current reaching the spinal nerve would likely be insufficient to cause depolarization. The same effect on current density is observed when using saline rather than dextrose during stimulator-guided peripheral nerve block. ${ }^{24,25}$ In other words, our findings suggest that the EST acts at the level of the spinal nerve which runs its course through the epidural space; however, further study will be needed to confirm this hypothesis. Nevertheless, the Figure summarizes an algorithm that can be used during CSE to help determine the location of the catheter tip and select the appropriate anesthetic dose based on location. It is important to interpret this algorithm with caution in light of the aforementioned limitations of EST results.

In this study, we used a double-segment CSE technique because we needed to have a control value for epidural stimulation before injection of local anesthetic. The double-segment technique was the only way possible to achieve this with our currently available equipment. Although some institutions may have moved away from using a double-segment technique, we find it to be a useful technique in surgical procedures where it is important to ensure that the catheter is secure before injecting the spinal component, especially when hyperbaric solutions are used. In this particular case, the technique was dictated by the study design. Another limitation of this study is its small sample size. Further research is needed since, at the present time, the EST cannot provide the information that can decidedly determine catheter positioning in situations of intrathecal injection of local anesthetic.

In summary, our results show that an intrathecal injection of a low dose of bupivacaine does not affect MTC if the catheter is placed in the epidural space. Nevertheless, injection of low-dose bupivacaine affects the MTC when the catheter is placed intrathecally. Our findings also confirm that the EST can help to determine the position of the epidural catheter prior to injection of the test dose.

Acknowledgement The authors thank Dr. Gareth Corry for assistance with manuscript preparation. Dr. Tsui is supported by a Clinical Scholar Award from the Alberta Heritage Foundation for Medical Research (AHFMR). Dr. Tsui's research is supported by the Canadian Anesthesia Research Foundation.

Conflicts of interest None declared.

\section{References}

1. Casati A, D’Ambrosio A, De Negri P, Fanelli G, Tagariello V, Tarantino $F$. A clinical comparison between needle-throughneedle and double-segment techniques for combined spinal and epidural anesthesia. Reg Anesth Pain Med 1998; 23: 390-4. 
2. Collis RE, Baxandall ML, Srikantharajah ID, Edge G, Kadim $M Y$, Morgan BM. Combined spinal epidural analgesia with ability to walk throughout labour. Lancet 1993; 341: 767-8.

3. Eldor J. Combined spinal-extradural anaesthesia in obstetrics. Br J Anaesth 1992; 68: 634-5.

4. Felsby $S$, Juelsgaard P. Combined spinal and epidural anesthesia. Anesth Analg 1995; 80: 821-6.

5. Lyons G, Macdonald $R$, Mikl B. Combined epidural/spinal anaesthesia for caesarean section. Through the needle or in separate spaces? Anaesthesia 1992; 47: 199-201.

6. Abdallah FW, Chan VW. From the Journal archives: Practical applicability of the epidural electrical stimulation test. Can J Anesth 2014; 61: 881-5.

7. Tsui BC, Gupta S, Finucane B. Confirmation of epidural catheter placement using nerve stimulation. Can J Anesth 1998; 45: 640-4.

8. de Medicis E, Tetrault JP, Martin R, Robichaud R, Laroche L. A prospective comparative study of two indirect methods for confirming the localization of an epidural catheter for postoperative analgesia. Anesth Analg 2005; 101: 1830-3.

9. Goobie SM, Montgomery CJ, Basu R, et al. Confirmation of direct epidural catheter placement using nerve stimulation in pediatric anesthesia. Anesth Analg 2003; 97: 984-8.

10. Hayatsu K, Tomita M, Fujihara H, et al. The placement of the epidural catheter at the predicted site by electrical stimulation test. Anesth Analg 2001; 93: 1035-9.

11. McAuliffe NA, Pickworth S, Direnna $T$, Hong A. Electrophysiological stimulation (Tsui test) is feasible for epidural catheter positioning in adults with chronic back pain: a cohort study. Can J Anesth 2013; 60: 976-81.

12. Morley-Forster PK, Abotaiban A, Ganapathy S, Moulin DE, Leung A, Tsui B. Targeted thoracic epidural blood patch placed under electrical stimulation guidance (Tsui test). Can J Anesth 2006; 53: 375-9.

13. Prusinkiewicz $C$, Lang $S$, Tsui BC. Lateral cervical epidural catheter placement using nerve stimulation for continuous unilateral upper extremity analgesia following a failed continuous peripheral nerve block. Acta Anaesthesiol Scand 2005; 49: 579-82.

14. Tamai H, Sawamura S, Kanamori Y, Takeda K, Chinzei M, Hanaoka $K$. Thoracic epidural catheter insertion using the caudal approach assisted with an electrical nerve stimulator in young children. Reg Anesth Pain Med 2004; 29: 92-5.

15. Tsui BC, Gupta S, Finucane B. Determination of epidural catheter placement using nerve stimulation in obstetric patients. Reg Anesth Pain Med 1999; 24: 17-23.

16. Tsui BC, Gupta S, Emery D, Finucane B. Detection of subdural placement of epidural catheter using nerve stimulation. Can J Anesth 2000; 47: 471-3.

17. Tsui BC, Usher A, Kulkarni PR, Scott SL. Thoracic epidural catheters via the caudal and lumbar approaches using styletted multiple port catheters in pediatric patients: a report of three cases. Acta Anaesthesiol Scand 2006; 50: 514-7.

18. Tsui BC, Bury J, Bouliane M, Ganapathy S. Cervical epidural analgesia via a thoracic approach using nerve-stimulation guidance in adult patients undergoing total shoulder replacement surgery. Acta Anaesthesiol Scand 2007; 51: 255-60.

19. Margarido CB, Dlacic A, Balki M, Furtado L, Carvalho JC. The epidural electric stimulation test does not predict local anesthetic spread or consumption in labour epidural analgesia. Can J Anesth 2013; 60: 393-8.

20. Standring $S$. Gray's Anatomy, $40^{\text {th }}$ Edition - The Anatomical Basis of Clinical Practice. London: Churchill Livingstone; 2008.

21. Sutherland MA, Viscomi CM, Dominick TS, Anderson EL. Minimum current requirements for epidural stimulation test confirmation of epidural and intrathecal catheter placement. Reg Anesth Pain Med 2009; 34: 575-7.

22. Quan D, Bird SJ. Nerve conduction studies and electromyography in the evaluation of peripheral nerve injuries. UPOJ 1999; 12: 45-51.

23. Tsui BC. Epidural stimulation test criteria. Anesth Analg 2006; 103: 775-6.

24. Tsui BC, Wagner A, Finucane B. Electrophysiologic effect of injectates on peripheral nerve stimulation. Reg Anesth Pain Med 2004; 29: 189-93.

25. Tsui BC, Kropelin B, Ganapathy S, Finucane B. Dextrose $5 \%$ in water: fluid medium for maintaining electrical stimulation of peripheral nerves during stimulating catheter placement. Acta Anaesthesiol Scand 2005; 49: 1562-5. 